\title{
Genome analysis of Yucatan miniature pigs to assess their potential as biomedical model animals
}

\author{
Dae-Jin Kwon ${ }^{1}$, Yeong-Sup Lee ${ }^{2}$, Donghyun Shin ${ }^{2}$, Kyeong-Hye Won ${ }^{2}$, and Ki-Duk Song ${ }^{1,2,3, *}$
}

* Corresponding Author: Ki-Duk Song Tel: +82-63-219-5523, Fax: +82-63-270-5937,

E-mail: kiduk.song@gmail.com

${ }^{1}$ International Agricultural Development and Cooperation Center, Chonbuk National University, Jeonju 54896, Korea

2 Department of Animal Biotechnology, Chonbuk

National University, Jeonju 54896, Korea

${ }^{3}$ The Animal Molecular Genetics and Breeding Center,

Chonbuk National University, Jeonju 54896, Korea

ORCID

Dae-Jin Kwon

https://orcid.org/0000-0002-9512-2283

Yeong-Sup Lee

https://orcid.org/0000-0001-9267-0850

Donghyun Shin

https://orcid.org/0000-0002-0819-0553

Kyeong-Hye Won

https://orcid.org/0000-0001-8112-2840

Ki-Duk Song

https://orcid.org/0000-0003-2827-0873

Submitted Feb 26, 2018; Revised Apr 24, 2018; Accepted May 29, 2018
Objective: Pigs share many physiological, anatomical and genomic similarities with humans, which make them suitable models for biomedical researches. Understanding the genetic status of Yucatan miniature pigs (YMPs) and their association with human diseases will help to assess their potential as biomedical model animals. This study was performed to identify non-synonymous single nucleotide polymorphisms (nsSNPs) in selective sweep regions of the genome of YMPs and present the genetic nsSNP distributions that are potentially associated with disease occurrence in humans.

Methods: nsSNPs in whole genome resequencing data from 12 YMPs were identified and annotated to predict their possible effects on protein function. Sorting intolerant from tolerant (SIFT) and polymorphism phenotyping v2 analyses were used, and gene ontology (GO) network and Kyoto encyclopedia of genes and genomes (KEGG) pathway analyses were performed.

Results: The results showed that 8,462 genes, encompassing 72,067 nsSNPs were identified, and 118 nsSNPs in 46 genes were predicted as deleterious. GO network analysis classified 13 genes into $5 \mathrm{GO}$ terms $(\mathrm{p}<0.05)$ that were associated with kidney development and metabolic processes. Seven genes encompassing nsSNPs were classified into the term associated with Alzheimer's disease by referencing the genetic association database. The KEGG pathway analysis identified only one significantly enriched pathway $(\mathrm{p}<0.05)$, hsa04080: Neuroactive ligand-receptor interaction, among the transcripts.

Conclusion: The number of deleterious nsSNPs in YMPs was identified and then these variants-containing genes in YMPs data were adopted as the putative human diseases-related genes. The results revealed that many genes encompassing nsSNPs in YMPs were related to the various human genes which are potentially associated with kidney development and metabolic processes as well as human disease occurrence.

Keywords: Yucatan Miniature Pigs; Functional Annotation; Next Generation Sequencing; Non-synonymous Single Nucleotide Polymorphisms; Genetic Association Database

\section{INTRODUCTION}

Recently, pigs have been used in biomedical research as they share many physiological and anatomical similarities with humans. In addition, pigs are evolutionarily closer to humans than mice [1]. By generating shotgun sequence data from the pig genome, almost all ultra-conserved elements in the human genome can also be detected in pigs [1]. However, conventional pigs are much larger ( $>300 \mathrm{~kg}$ adult size) than other experimental animals (mice, rats, and dogs), which make them harder to handle. In comparison, Yucatan pigs (YMPs) are smaller than conventional pigs, with a mean body weight of $31 \mathrm{~kg}$ at 8 months and $83 \mathrm{~kg}$ at 24 months [2]. Furthermore, they have distinct traits, such as gentleness, intelligence, resistance to disease, and relative lack of odor, which make them more desirable to use for biomedical research purposes $[3,4]$. 
Genetic variations are classified by single nucleotide polymorphisms (SNPs), which occur from small insertions, deletions, conversions, and rearrangements. SNPs constitute almost $90 \%$ of the genetic variation in the human genome [5]. Most SNPs are stable and not deleterious to organisms, probably as most are located in intergenic spacers. However, non-synonymous SNPs (nsSNPs) could influence promoter activity or DNA, which is likely to affect the structure and function of proteins [6]. For instance, multiple nsSNPs in innate immunity genes can affect the susceptibility to infection, as well as the development of inflammatory disorders and autoimmune diseases [7,8]. Thus, nsSNPs are good candidates for studying disease-modifying alleles. Notably, nsSNPs that involve amino acid substitutions and cause structural and functional changes in proteins that are related to biological functions are reflective of breed characteristics $[9,10]$.

Understanding the genetic status of YMPs and their association with human diseases will help to assess their potential as biomedical model animals. In the present study, whole genome sequencing data of YMPs was integrated and reanalyzed to identify genomic variants, and nsSNPs in selective sweep regions were identified. Using nsSNPs from YMPs, we first found deleterious nsSNPs in YMPs and then adopted these variants-containing genes in YMPs data as the putative human diseases-related genes.

\section{MATERIALS AND METHODS}

Sample preparation and whole genome sequencing

A whole genome sequence dataset from 12 YMPs was retrieved from the National Center for Biotechnology Information sequence read archive database (SRP047260). FastQC software (Available from: http://wwwbioinformaticsbabrahamacuk/ projects/fastqc) was used to perform a quality check on raw sequence data. Trimmomatic-0.32 [11] was used to remove potential adapter sequences before sequence alignment, followed mapping of paired-end reads to the pig reference genome (Sscrofa 10.2.75) that was obtained from the Ensembl database using Bowtie2 [12] with default settings. Picard tools (http:// broadinstitute.github.io/picard/), SAMtools [13], and Genome Analysis Toolkit (GATK) [14] were used for downstream processing and variant calling. "CreateSequenceDictionary" and "MarkDuplicates" Picard command-line tools were used to read reference FASTA sequences to write bam files with only a sequence dictionary and to filter potential PCR duplicates, respectively. Index files for the reference and bam files were created using SAMtools. Local realignment of sequence reads to correct misalignment was performed to remove small insertions and deletions using GATK "Realigner-TargetCreator" and "IndelRealigner" arguments. In addition, base quality score recalibration was performed to obtain accurate quality scores and to correct the variation in quality from machine cycle and sequence context. For variant calling, GATK "UnifiedGenotyper" and "SelectVariants" arguments were used with the following filtering criteria: all variants with i) a Phredscaled quality score of $<30$; ii) read depth $<5$; iii) MQ0 (total count across all samples of mapping quality zero reads) $>4$; iv) Phred-scaled p-values of more than 200 using Fisher's exact test were filtered out to reduce false-positive calls due to strand bias. Additionally, all filtered SNPs on autosomes (a total of $26,240,429$ SNPs) were annotated using a SNP annotation tool, SnpEff version 4.1a and the Ensemble Sus scrofa gene set version 75 (Sscrofa10.2.75). The number of genes was 27,498.

Identification of nsSNPs in YMP selective sweep regions A previous study identified a total of 390 selective sweep regions and 429 associated genes in YMPs from three comparative analyses via a cross-population extended haplotype homozygosity (XP-EHH) analysis: 164 regions (184 genes), 137 regions (146 genes), and 181 regions (196 genes) in Duroc, Landrace, and Yorkshire breeds, respectively [2]. Among the 429 genes, 269 genes overlapped with nsSNP-containing genes. Thus, 269 genes containing nsSNPs were further analyzed using sorting intolerant from tolerant (SIFT) and polymorphism phenotyping V2 (Polyphen-2) to survey whether these nsSNPs were deleterious.

\section{Prediction of deleterious amino-acid substitutions in YMP nsSNPs}

We predicted the functional effects of nsSNPs using the following in silico algorithms: SIFT [15] and Polyphen-2 [16]. The SIFT uses sequence homology to predict the effects of amino acid substitution on protein functions. We performed nsSNP-based sequence homology tests to identify important amino acid substitutions that might affect biological functions by structurally modifying proteins. A SIFT score of $<0.05$ indicated that the SNP was deleterious and could strongly affect protein function. Moreover, we performed a PolyPhen-2 (version 2.2.2) analysis using specific empirical rules to predict the effects of amino acid substitutions on the structures and functions of proteins, using amino acid sequences containing target SNPs from the Ensemble database. We then tested proteins with 1,726 nsSNPs in YMP selective sweep regions using Polyphen-2. Tested nsSNPs were classified as "probably damaging", "possibly damaging", or "benign" if they received Polyphen-2 scores (range: 0 to 1 ) of $>0.95,0.5$ to 0.95 , or $<0.5$, respectively. In this study, we predicted that "probably damaging" and "possibly damaging" SNPs would likely affect protein functions.

\section{Bioinformatics analysis}

Genes encompassing deleterious nsSNPs in YMPs were functionally annotated and classified using the Database for Annotation, Visualization, and Integrated Discovery (DAVID) 
Functional Annotation Tool (http://david.abcc.ncifcrf.gov/), which provides integrated solutions for the anno $\neg$ tation and analyses of genome-scale datasets that are derived from highthroughput technologies. The analyzed genes were considered to be able to apply to the human genes. Thus the database used in DAVID was human. We considered that gene ontology (GO) of deleterious nsSNPs and those-containing genes in YMPs might be important in human diseases, also. Additionally, pathways were elucidated according to the Kyoto encyclopedia of genes and genomes (KEGG) and the related diseases were analyzed based on the genetic association database (GAD).

\section{RESULTS}

DNA sequencing data preprocessing and genetic variant calling

We extracted a total of $28,960,830$ SNPs from the whole-genome sequences of 12 YMPs and annotated all extracted SNPs using SnpEff version 4.1a [17]. The result was 8,461 genes, encompassing 72,067 nsSNPs (Figure 1). As shown in Figure 1A, the number of known variants was 19,717,922 (68.08\%) and the number of novel variants was 9,242,908 (31.92\%). In Figure $1 \mathrm{~B}$, the number of nsSNPs was 72,607 and the number of synonymous SNPs was 113,426 . Thus, 72,607 nsSNPs were analyzed further to identify disease-related genes. Since YMPs are important in the investigation of human diseases, we used nsSNPs-containing genes of YMPs (8,461 genes). Figure 1C shows the number of nsSNPs per chromosome. The highest number of nsSNPs was on chromosome $2(7,595)$, while the lowest number of nsSNPs was on chromosome $11(1,202)(\mathrm{mi}-$ tochondrial genomes were not considered).

\section{Selective sweep genes and genes with nsSNPs}

From the 8,461 nsSNP-encompassing genes, we selected 269 genes that overlapped with the 429 selective sweep genes in the previous study [2]. Additionally, we conducted comprehensive in silico nsSNP analysis on YMPs and predicted the possible effects of the nsSNPs on protein structure and function using SIFT and Polyphen-2. We identified that 118 nsSNPs of 46 genes might be deleterious (Table 1). The total number of SNPs analyzed was 1,726. In the SIFT analysis, there were 224 deleterious SNPs and the number of deleterious SNPs with low confidence was 45 . In the Polyphen 2 analysis, there were 187 probably damaging nsSNPs, 147 possibly damaging nsSNPs, and 663 benign nsSNPs, as shown in Table 1 . We selected deleterious nsSNPs in SIFT and probably damaging nsSNPs in Polyphen2 (48 in total) for GO analysis. The gene catalogue was retrieved from the ensemble website (www. ensembl.org).
A

SNP Calling

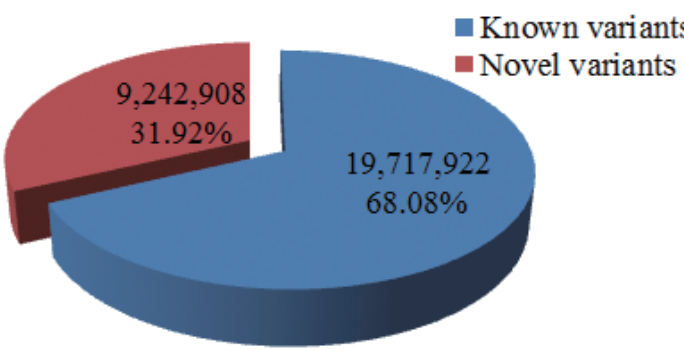

C

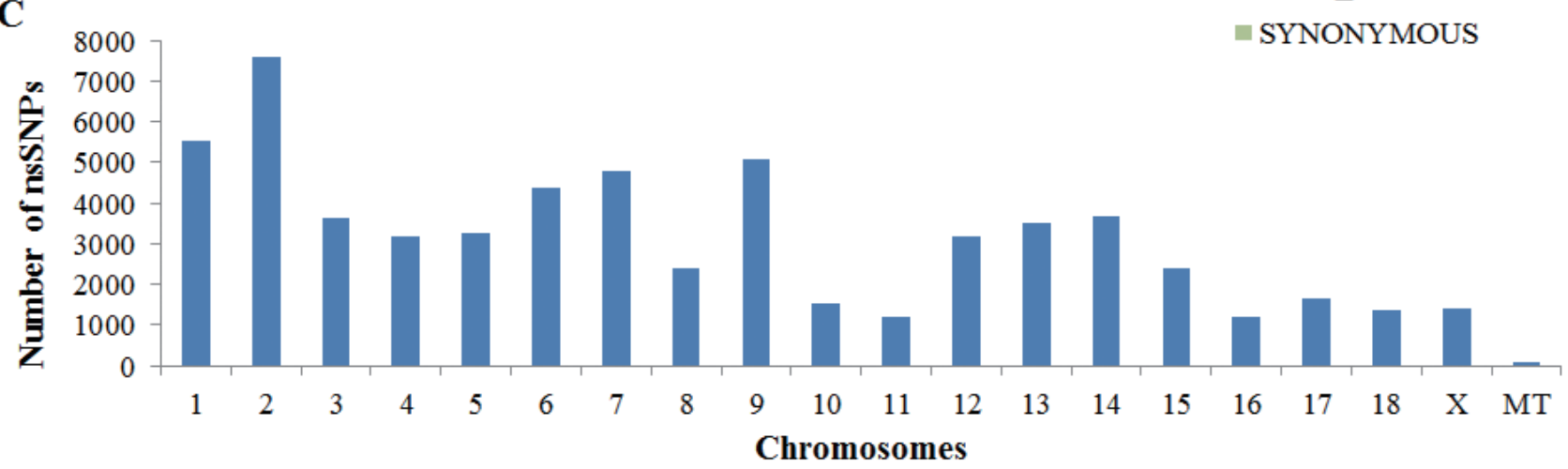

- NON_SYNONYMOUS

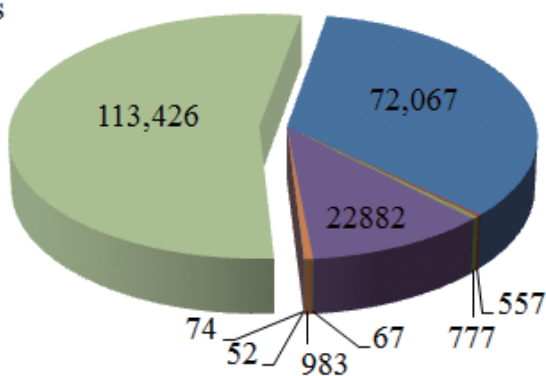

- SPLICE_ACCEPTOR

- SPLICE_DONOR

- SPLICE_REGION

- START_LOST

- STOP_GAINED

- STOP_LOST

- STOP RETAINED

- SYNONYMOUS
Chromosomes

Figure 1. Overview of the identified single nucleotide polymorphisms (SNPs) from Yucatan miniature pigs. (A) The number of the known rs-tagged variants and novel variants. (B) The SNP categories in SnpEff analysis. (C) The number of non-synonymous SNPs across chromosomes. 
Table 1. Summarization of non-synonymous single amino acid variation in genes of Yucatan miniature pigs selective sweep using SIFT and Polyphen-2

\begin{tabular}{|c|c|c|c|c|c|c|}
\hline \multirow{2}{*}{ Item } & & \multicolumn{5}{|c|}{ SIFT } \\
\hline & & Deleterious & Deleterious low confidence & Tolerated & Tolerated low confidence & Total \\
\hline \multirow[t]{5}{*}{ Polyphen-2 } & Benign & 58 & 10 & 536 & 59 & 663 \\
\hline & Possibly damaging & 47 & 7 & 81 & 12 & 147 \\
\hline & Probably damaging & 118 & 7 & 62 & 0 & 187 \\
\hline & Unknown & 1 & 21 & 16 & 21 & 59 \\
\hline & Total & 224 & 45 & 695 & 92 & 1,056 \\
\hline
\end{tabular}

SIFT, sorting intolerant from tolerant; Polyphen-2, polymorphism phenotyping v2.

\section{Functional classification}

GO analysis was used to analyze the biological processes (BP, level 3) and KEGG pathways associated with the functioning of the genes that encompassed nsSNPs in YMPs (Table 2). GO network analysis classified 13 genes, i.e., catalytic subunit of the oligosaccharyltransferase complex, keratocan, glutamate metabotropic receptor 8 (GRM8), NADH: ubiquinone oxidoreductase subunit $\mathrm{B} 9$, dopamine receptor $\mathrm{D} 5$ (DRD5), platelet derived growth factor receptor beta (PDGFRB), MACRO domain containing 1 , nuclear receptor corepressor 1 (NCOR1), follicle stimulating hormone receptor (FSHR), N-acylsphingosine amidohydrolase 1 (ASAH1), MTSS1, I-BAR domain containing (MTSS1), glutamyl aminopeptidase (ENPEP), and uroplakin 3A, encompassing 118 nsSNPs, into 5 GO terms $(\mathrm{p}<0.05)$ that were associated with kidney development and metabolic processes.

We identified the relationship between these genes and diseases based on the GAD and confirmed several genes with the associated disease categories. Five (ankyrin1 [ANK1], receptor tyrosine kinase like orphan receptor 1 [ROR1], early endosome antigen 1 [EEA1], programmed cell death 6 interacting protein $[P D C D 6 I P], \mathrm{BMP} /$ retinoic acid inducible neural specific 3 [BRINP3]), 7 (amyloid precursor protein [APP], PDGFRB, membrane palmitoylated protein 7 , PHD finger protein 12 , glutamate ionotropic receptor nmda type subunit 3A [GRIN3A], FSHR, arginyltransferase 1 [ATE1]), and 12 (APP, small G protein signaling modulator 1 [SGSM1], ROR1, PDGFRB, GRIN3A, seizure related 6 homolog [SEZ6L], NCOR1, FSHR, solute carrier family 22 member 2 [SLC22A2], TBC1 domain family member 32 [TBC1D32], OCA2 melanosomal transmembrane protein [OCA2], ASAH1) genes were associated with low-density lipoprotein (LDL) cholesterol, Alzheimer's disease, and type 2 diabetes, respectively (Table 3 ).

KEGG pathway analysis identified 2 enriched pathways, hsa04080: Neuroactive ligand-receptor interaction (GRM8, DRD5, GRIN3A, FSHR) and hsa04024: cAMP signaling path-

Table 2. Gene ontology (GO) classification of genes from non-synonymous single nucleotide polymorphisms data in Yucatan miniature pigs

\begin{tabular}{|c|c|c|c|}
\hline Term & Count & $\mathrm{p}$ value & Genes \\
\hline G0:1901135 carbohydrate derivative metabolic process & 10 & 0.004652 & $\begin{array}{l}\text { STT3B, KERA, GRM8, NDUFB9, DRD5, PDGFRB, MACROD1, NCOR1, } \\
\text { FSHR, ASAH1 }\end{array}$ \\
\hline G0:0032835 glomerulus development & 3 & 0.012017 & MTSS1, PDGFRB, ENPEP \\
\hline G0:0072102 glomerulus morphogenesis & 2 & 0.020935 & MTSS1, PDGFRB \\
\hline G0:0001822 kidney development & 4 & 0.033262 & MTSS1, PDGFRB, ENPEP, UPK3A \\
\hline $\begin{array}{l}\text { GO:0055086 nucleobase-containing small molecule } \\
\text { metabolic process }\end{array}$ & 6 & 0.041847 & GRM8, NDUFB9, DRD5, MACROD1, NCOR1, FSHR \\
\hline GO:0072006 nephron development & 3 & 0.051382 & MTSS1, PDGFRB, ENPEP \\
\hline G0:0048513 animal organ development & 14 & 0.056305 & $\begin{array}{l}\text { TG, MTSS1, KERA, ENPEP, UPK3A, SEZ6L, FSHR, APP, CC2D2A, ROR1, } \\
\text { PDGFRB, TBC1D32, KAT6A, SCN10A }\end{array}$ \\
\hline G0:0007617 mating behavior & 2 & 0.056545 & APP, DRD5 \\
\hline G0:1901564 organonitrogen compound metabolic process & 11 & 0.060428 & $\begin{array}{l}\text { TG, KERA, GRM8, NDUFB9, DRD5, PDGFRB, ENPEP, MACROD1, } \\
\text { NCOR1, FSHR, ASAH1 }\end{array}$ \\
\hline G0:0021915 neural tube development & 3 & 0.067419 & SHROOM3, CC2D2A, TBC1D32 \\
\hline GO:0019098 reproductive behavior & 2 & 0.076322 & APP, DRD5 \\
\hline G0:0048731 system development & 17 & 0.078392 & $\begin{array}{l}\text { TG, MTSS1, SHROOM3, KERA, ENPEP, UPK3A, GRIN3A, SEZ6L, FSHR, } \\
\text { APP, CC2D2A, ROR1, PDGFRB, BRINP3, TBC1D32, SCN10A, KAT6A }\end{array}$ \\
\hline GO:0007017 microtubule-based process & 5 & 0.079313 & $A P P, C C 2 D 2 A, N I N L, P D C D 6 I P, N C O R 1$ \\
\hline G0:0034405 response to fluid shear stress & 2 & 0.086058 & MTSS1, PDGFRB \\
\hline GO:0007618 mating & 2 & 0.093294 & APP, DRD5 \\
\hline G0:0044765 single-organism transport & 14 & 0.09973 & $\begin{array}{l}\text { TG, SERGEF, LRRC32, SLC22A23, UPK3A, GRIN3A, APP, ANK1, } \\
\text { PDGFRB, PDCDGIP, NCOR1, SLC22A2, OCA2, SCN10A }\end{array}$ \\
\hline
\end{tabular}


Table 3. The disease categories provided by genetic association database and disease-related genes from non-synonymous single nucleotide polymorphisms data in Yucatan miniature pigs

\begin{tabular}{|c|c|c|c|}
\hline Term & Count & $p$ value & Genes \\
\hline Tobacco use disorder & 24 & $1.10 \mathrm{E}-05$ & $\begin{array}{l}\text { TG, MTSS1, SVEP1, SHROOM3, SLC22A23, MPP7, ENPEP, SEZ6L, GRIN3A, ASAH1, ATE1, STT3B, } \\
\text { APP, SGSM1, ANK1, GRM8, ROR1, DCLK3, PDCD6IP, BRINP3, USP43, TBC1D32, OCA2, SCN10A }\end{array}$ \\
\hline Hip & 6 & 0.005931 & SHROOM3, ANK1, EFCAB6, PDCD6IP, BRINP3, OCA2 \\
\hline Cholesterol & 6 & 0.011794 & MTSS1, ROR1, EEA1, ENPEP, BRINP3, FSHR \\
\hline Electrocardiography & 4 & 0.013432 & GRM8, MPP7, BRINP3, SCN10A \\
\hline Echocardiography & 5 & 0.035536 & MTSS1, MPP7, SYNPO2, BRINP3, TBC1D32 \\
\hline Body mass index & 5 & 0.068273 & GRM8, MPP7, USP43, SLC22A2, TBC1D32 \\
\hline Weight gain & 3 & 0.068864 & GRM8, DRD5, GRIN3A \\
\hline Cholesterol, low-density lipoprotein & 5 & 0.069947 & ANK1, ROR1, EEA1, PDCD6IP, BRINP3 \\
\hline Alcoholism & 5 & 0.081344 & MTSS1, GRM8, SYNPO2, ENPEP, SEZ6L \\
\hline Alzheimer's disease & 7 & 0.083627 & APP, PDGFRB, MPP7, PHF12, GRIN3A, FSHR, ATE1 \\
\hline Type 2 diabetes |edema| rosiglitazone & 12 & 0.086573 & APP, SGSM1, ROR1, PDGFRB, GRIN3A, SEZ6L, NCOR1, FSHR, SLC22A2, TBC1D32, OCA2, ASAH1 \\
\hline
\end{tabular}

way (DRD5, GRIN3A, FSHR) (Table 4).

\section{DISCUSSION}

Animal models for human disease, especially mouse models, have been used to understand the mechanisms involved in human hematopoiesis, innate and adaptive immunity, autoimmunity, infectious diseases, cancer biology, and regenerative medicine [18]. While many studies of human diseases have used mice and provided numerous insights into the biology of cancer and regenerative medicine, experiments using inbred lines of mice are limited in their translation of results to humans [19]. By comparison, pigs share many physiological, anatomical, and genomic similarities with humans. Therefore, information on the genetic status of pigs could be of greater help in the understanding of complex genetic disease variation in humans. Here, we identified nsSNPs in YMPs selective sweep regions and represented genetic nsSNP distributions that are potentially associated with disease occurrence in humans.

GO network analysis showed that 13 of 46 genes, encompassing 118 nsSNPs, were associated with kidney development and metabolic processes. Furthermore, we identified disease terms by consulting with the GAD and identified 5 genes, i.e., ANK1, ROR1, EEA1, PDCD6IP, and BRINP3 that were associated with LDL cholesterol. LDL cholesterol is known to be a key causal factor of atherosclerotic vascular disease, especially coronary heart disease [20]. Basically, elevation of LDL cholesterol in plasma concentrations is due to single variants in the coding region of genes for the LDL receptor, apolipoprotein $\mathrm{B}$, and proprotein convertase subtilisin/kexin type 9 [21]. YMPs exhibit some of the metabolic lesions observed in obese humans. Thus, insulin resistance, mild diabetes, and atherosclerotic lesions could be induced by feeding YMPs with a high-fat/high-sucrose diet [22]. In a previous study, hyperglycemia occurred in pigs that became diabetic after insulinproducing cells were destroyed, and there were no adverse effects on the kidney and liver functions [23]. Further, the lipoprotein profile in diabetic pigs was similar to that found in human diabetic patients, which suggests that pigs may be an appropriate animal model for studying metabolic disorders as they can be used to uncover the pathogenic components of the diabetic milieu or the mechanisms of atherosclerosis [24].

The genes APP, SGSM1, ROR1, PDGFRB, GRIN3A, SEZ6L, NCOR1, FSHR, SLC22A2, TBC1D32, OCA2, and ASAH1, which also encompassed nsSNPs, were classified into Alzheimer's disease after reference to the GAD. Alzheimer's disease is a chronic neurodegenerative disease involving loss of memory and bodily functions and ultimately causes death. Alzheimer's disease is caused by a mutation in the APP gene, or in the presenilin 1 (PSEN1) and 2 (PSEN2) genes. It is now clear that mutations in all these genes alter the production of the amyloid $\beta$-peptide $(\mathrm{A} \beta)$ fragment of APP, and trigger the formation of amyloid plaques that eventually result in neuronal dysfunction [25]. Transgenic mice with a mutated human APP gene that were designed to promote $A \beta$ accumulation and deposition formed neuronal plaques; however, neuronal loss did not occur in the hippocampus or association cortex, which is characteristic of Alzheimer's disease [26]. As pigs are evolutionarily closer to humans, they can be used to generate transgenic pigs using a splice variant of the human APP that carries an Alzheimer's disease-causing dominant mutation [26]. Although transgenic pigs showed high levels of trans-

Table 4. Kyoto encyclopedia of genes and genomes pathway analysis of genes from non-synonymous single nucleotide polymorphisms data in Yucatan miniature pigs

\begin{tabular}{lccc}
\hline Term & Count & p value & Genes \\
\hline hsa04080:Neuroactive ligand-receptor interaction & 4 & 0.033281 & GRM8, DRD5, GRIN3A, FSHR \\
hsa04024:CAMP signaling pathway & 3 & 0.092596 & DRD5, GRIN3A, FSHR \\
\hline
\end{tabular}


gene expression, including high levels of the corresponding protein in brain tissues, $A \beta$ accumulation and deposition did not occur in this tissue [27]. APP processing in guinea pigs is identical to that in humans [28]. Nonetheless, there is no appropriate pig model for Alzheimer's disease. Therefore, exploiting data for Alzheimer's disease-related genes that encompass nsSNPs in YMPs may be useful for deciphering the mechanisms of Alzheimer's disease and improving a therapeutic design.

\section{CONCLUSION}

The present study surveyed the nsSNPs in YMPs and analyzed human GO using DAVID. The number of deleterious nsSNPs in YMPs was identified and then these variants-containing genes in YMPs data were adopted as the putative human diseases-related genes. The results revealed that many genes encompassing nsSNPs in YMPs were related to the various human genes which are potentially associated with kidney development and metabolic processes as well as the occurrence of human disease. Currently, the generation of transgenic pigs with single- or multiple-gene modifications, as well as chromosomal translocations, for use as biomedical models of human disease is possible using precise genetic engineering techniques [29]. With these techniques, a deeper insight into the genetic relationship between human and pig genes could help to elucidate the complex genetic disease variation-susceptibility relationship and facilitate the application of YMPs as a good candidate for a large animal model for human diseases.

\section{CONFLICT OF INTEREST}

We certify that there is no conflict of interest with any financial organization regarding the material discussed in the manuscript.

\section{ACKNOWLEDGMENTS}

This study was supported by a grant from the Next-Generation BioGreen 21 Program (No. PJ01110901, PJ01315101, PJ01337702), Rural Development Administration, Republic of Korea. The authors are grateful to this organization.

\section{REFERENCES}

1. Wernersson R, Schierup MH, Jørgensen FG, et al. Pigs in sequence space: a $0.66 \mathrm{X}$ coverage pig genome survey based on shotgun sequencing. BMC Genomics 2005;6:70.

2. Kim H, Song KD, Kim HJ, et al. Exploring the genetic signature of body size in Yucatan miniature pig. PLoS One 2015; 10:e0121732.
3. Panepinto L, Phillips R. The Yucatan miniature pig: characterization and utilization in biomedical research. Lab Anim Sci 1986;36:344-7.

4. Smith DM, Martens GW, Ho CS, Asbury JM. DNA sequence based typing of swine leukocyte antigens in Yucatan miniature pigs. Xenotransplantation 2005;12:481-8.

5. Groenen MA, Archibald AL, Uenishi H, et al. Analyses of pig genomes provide insight into porcine demography and evolution. Nature 2012;491:393-8.

6. Radivojac P, Vacic V, Haynes C, et al. Identification, analysis, and prediction of protein ubiquitination sites. Proteins 2010; 78:365-80.

7. Azad AK, Sadee W, Schlesinger LS. Innate immune gene polymorphisms in tuberculosis. Infect Immun 2012:80:3343-59.

8. Netea MG, Wijmenga C, O'Neill LA. Genetic variation in Toll-like receptors and disease susceptibility. Nat Immunol 2012;13:535-42.

9. Noreen M, Arshad M. Association of TLR1, TLR2, TLR4, TLR6, and TIRAP polymorphisms with disease susceptibility. Immunol Res 2015;62:234-52.

10. Ghosh M, Sodhi SS, Sharma N, et al. An integrated in silico approach for functional and structural impact of non- synonymous SNPs in the MYH1 gene in Jeju Native Pigs. BMC Genet 2016;17:35.

11. Bolger AM, Lohse M, Usadel B. Trimmomatic: a flexible trimmer for Illumina sequence data. Bioinformatics 2014;30:211420.

12.Langmead B, Salzberg SL. Fast gapped-read alignment with Bowtie 2. Nat Methods 2012;9:357-9.

13.Li H, Handsaker B, Wysoker A, et al. The Sequence Alignment/ Map format and SAMtools. Bioinformatics 2009;25:2078-9.

14. McKenna A, Hanna M, Banks E, et al. The Genome Analysis Toolkit: a MapReduce framework for analyzing next-generation DNA sequencing data. Genome Res 2010;20:1297-303.

15. Ng PC, Henikoff S. SIFT: Predicting amino acid changes that affect protein function. Nucleic Acids Res 2003;31:3812-4.

16. Adzhubei I, Jordan DM, Sunyaev SR. Predicting functional effect of human missense mutations using PolyPhen-2. Curr Protoc Hum Genet 2013;Chapter 7:Unit7 20.

17. Cingolani P, Platts A, Wang le L, et al. A program for annotating and predicting the effects of single nucleotide polymorphisms, SnpEff: SNPs in the genome of Drosophila melanogaster strain w1118; iso-2; iso-3. Fly (Austin) 2012;6:80-92.

18. Shultz LD, Ishikawa F, Greiner DL. Humanized mice in translational biomedical research. Nat Rev Immunol 2007;7:118-30.

19. Hunter KW. Mouse models of cancer: does the strain matter? Nat Rev Cancer 2012;12:144-9.

20. Babiak J, Rudel LL. Lipoproteins and atherosclerosis. Baillieres Clin Endocrinol Metab 1987;1:515-50.

21. Burnett JR, Hooper AJ. Common and rare gene variants affecting plasma LDL cholesterol. Clin Biochem Rev 2008;29:11-26. 22. Xi S, Yin W, Wang Z, et al. A minipig model of high-fat/high- 
sucrose diet-induced diabetes and atherosclerosis. Int J Clin Exp Pathol 2004;85:223-31.

23. Dixon JL, Stoops J, Parker J, et al. Dyslipidemia and vascular dysfunction in diabetic pigs fed an atherogenic diet. Arterioscler Thromb Vasc Biol 1999;19:2981-92.

24. Johansen T, Hansen HS, Richelsen B, Malmlöf K. The Obese Göttingen minipig as a model of the metabolic syndrome: dietary effects on obesity, insulin sensitivity, and growth hormone profile. Comp Med 2001;51:150-5.

25.Hardy J, Selkoe DJ. The amyloid hypothesis of Alzheimer's disease: progress and problems on the road to therapeutics. Science 2002;297:353-6.

26. Takeuchi A, Irizarry MC, Duff K, et al. Age-related amyloid $\beta$ deposition in transgenic mice overexpressing both Alzheimer mutant presenilin 1 and amyloid $\beta$ precursor protein Swedish mutant is not associated with global neuronal loss. Am J Pathol 2000;157:331-9.

27.Kragh PM, Nielsen AL, Li J, et al. Hemizygous minipigs produced by random gene insertion and handmade cloning express the Alzheimer's disease-causing dominant mutation APPsw. Transgenic Res 2009;18:545-58.

28. Beck M, Bigl V, Roßner S. Guinea pigs as a nontransgenic model for APP processing in vitro and in vivo. Neurochem Res 2003; 28:637-44.

29. Prather RS, Shen M, Dai Y. Genetically modified pigs for medicine and agriculture. Biotechnol Genet Eng Rev 2008;25:24565. 